\title{
Performance Evaluation for STBC OFDM-CDMA System over Multi-Path Fast Fading Channel with the Aid of Various Channel Coding Techniques
}

\author{
Nelly Muhammad Hussein ${ }^{*}$, Adel El-Sherif**, and Abd El-Wahab Fayez ${ }^{* * *}$
}

\begin{abstract}
In this paper, a mitigation method for minimizing the distortion caused by frequency selective fast fading is illustrated. The proposed system combines between two types of diversity schemes; frequency diversity represented by the orthogonal frequency division multiplexing (OFDM) scheme and antenna diversity represented by space time block coding (STBC) scheme these are for mitigating the distortion caused by frequency selective fading. The multiplexing technique proposed here is the code division multiple access (CDMA) scheme which is considered the solution for eliminating the distortion caused by fast fading. The main problem of the OFDM system is the high peak-to-average power ratio (PAR). In the proposed system, three techniques used for reducing the PAR are applied together. And in order to reduce the bit error rate caused by intersymbol interference (ISI) or multiple access interference (MAI) channel coding is applied which can make error detection and correction at the receiver and simulation results have shown that by applying any of channel coding techniques, the bit error rate (BER) has reached a satisfying level at a low signal-to-noise ratio (SNR).
\end{abstract}

Keywords: OFDM , CDMA, STBC, and PAR

\section{Introduction}

One of the main problems facing wireless transmission system is the distortion caused by fading. The worst type of fading channel is the frequency selective fast fading in which the spectrum of transmitted message signal is distorted as a result of frequency selective fading besides the time dispersion of the received symbols caused by fast fading resulting in inter symbol interference (ISI). The OFDM combined with STBC is considered an affective solution for mitigating the frequency selective problems.

The performance of OFDM systems with multiple transmit antennas is introduced by the aid of common preamble for channel parameters estimation at [3]. The main performance limitation in the OFDM system is that the transmitted signal has high peak-to-average power ration (PAR) which may cause the receiver amplifiers to be saturated leading to degradation in the receiver performance that appears in the rising of bit error rate level. In order to reduce

\footnotetext{
* Lecturer assistant, Modern Academy for Engineering and Technology, nelly_mohammed@yahoo.com

${ }^{* *}$ Prof., Modern Academy for Engineering and Technology adelsherif@yahoo.com.au

*** Prof., Faculty of Engineering - Cairo University afayez2000@yahoo.com
} 
the PAR, three techniques have been used in the proposed system; simplified symbol formatting, modified selective mapping approach (SLM) [6] and modified partial transmit sequence approach (PTS) [5] that are to be described in details in the coming subsections.

The main drawback of traditional techniques used for PAR reduction is the side information provided into the received sequence but the proposed schemes; SLM and PTS result in less side information and less complexity when compared to the original schemes. In order to reduce the BER level, one of the channel coding schemes could be used such as Hamming or convolutional coding which have the ability to make error detection and correction at the receiver. By applying any of the previous channel coding techniques very low BER value was obtained at relatively small SNR.

The contents of the coming sections are arranged as follows: Section 2 describes the STBC OFDM- CDMA transmitter model with the aid of channel coding. At section 3, the proposed channel model specifications are described in details. STBC OFDM-CDMA receiver will be described at section 4. Section 5 displays the simulation results using computer software which are displayed in form of set of curves and tables.

\section{STBC OFDM - CDMA Transmitter Model}

There are $\mathrm{K}$ users each transmits binary data sequence. Then one of digital modulation techniques is applied such as BPSK, QPSK, or DBSK. The modulated symbol of user $k$ is denoted by $D_{k}^{t}$ where $t$ is the signaling time instant. In case of BPSK and DBPSK $D_{k}^{t}=\{ \pm 1\}$

whereas, in QPSK modulation case, $D_{k}^{t}=\{ \pm 1, \pm j\}$. Each modulated symbol is spread by PN code uniquely identifying each user and the Walsh code is used here for that purpose. The PN code of user $k$ is denoted by $W_{k}$ with length of M chips. The symbol after spreading could be expressed as follows:

$$
\underline{S}_{k}^{t}=D_{k}^{t} \underline{W}_{k} \quad \ldots
$$

The Walsh code could be obtained by many methods but the simplest way is by using Hadamard matrix which is a square matrix of elements $\{ \pm 1\}$ and the cross-correlation between each two rows equals to zero therefore, each row can be a perfect PN code with the guarantee of no multiple access interference among users. Another advantage of the Walsh code is that when all transmitted symbols by the $\mathrm{K}$ users are summed, it will be destructive summation which means the PAR of the superimposed signal will be reduced [6]. The proposed model of the STBC ODFM - CDMA is displayed in Fig.1.

\subsection{Simplified Symbol Formatting}

As mentioned before, the main problem in the OFDM system is the relatively high PAR and before displaying the proposed methods for reducing the PAR, let's define the PAR first:

$$
P A R=\frac{\max _{0 \leq n \leq N-1}\left\{\left|s_{k, n}^{t}\right|^{2}\right\}}{E\left[\left|s_{k, n}^{t}\right|^{2}\right]} \quad \ldots
$$


where; $s_{k}$ is the time domain sequence constructed from $S_{k}^{t}$ by evaluating N-point inverse fast Fourier transform IFFT for $S_{k}^{t}$. In that sachem, the sequence denoted by $\underline{S}_{k}^{t}$ is divided into two halves; $\underline{S}_{k}^{t}[1]$ and $\underline{S}_{k}^{t}[2]$ where, the first halve is transmitted as it is whereas, the conjugate of the second halve will be emitted.

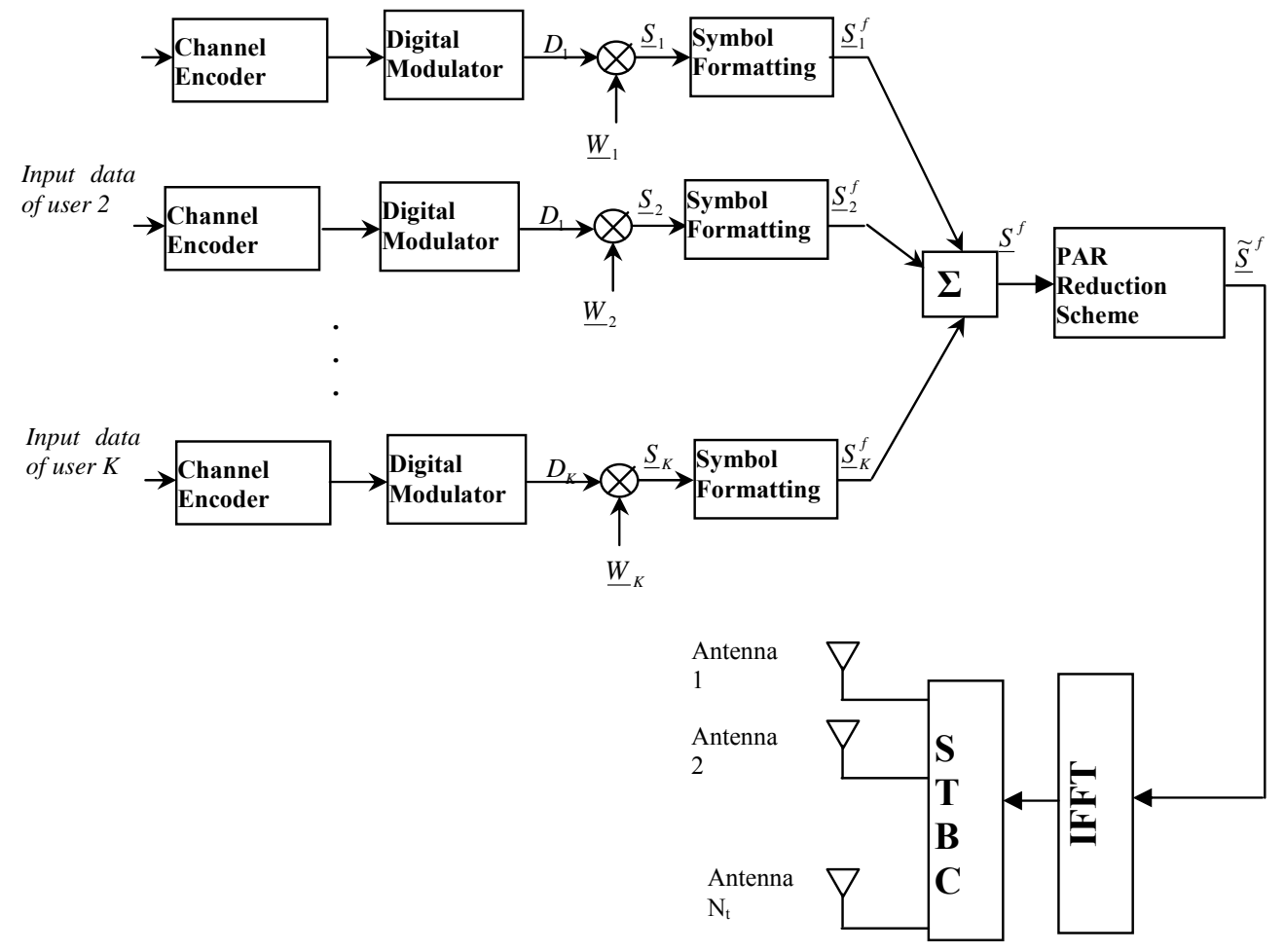

Fig. 1 STBC OFDM - CDMA Transmitter Model.

The previous steps could be summarized in the coming equation:

$$
\underline{S}_{k}^{f}=\left[\underline{S}_{k}[1] \underline{S}_{k}^{*}[2]\right] \quad \ldots
$$

Beginning from this point and forward, the parameter $t$ will be omitted for simplicity and since all the coming analysis refers to one time instant.

\subsection{Selective Mapping (SLM) Algorithm}

This algorithm is applied to the summated sequences from the $\mathrm{K}$ users given by:

$$
\underline{S}^{f}=\sum_{k=1}^{K} \underline{S}_{k}^{f} \quad \cdots
$$

In the traditional SLM scheme [6], [8] a set of complex vectors $P^{(g)}$ are first randomly generated each with size $N$ and the total number of vectors is $G$. The elements of those vectors 
are described as: $P^{(g)}=\{ \pm 1, \pm j\}$ and $1 \leq g \leq G$. Each vector is multiplied by the vector $\underline{S}^{f}$ giving a group of vectors $\left\{\underline{S}^{f}(g), 1 \leq g \leq G\right\}$ which could be represented as follows:

$$
S_{n}^{f}(g)=S_{n}^{f} \cdot P_{n}^{(g)}, 0 \leq n \leq N-1 \quad \ldots
$$

Then the PAR is calculated for all obtained vectors and the one with the lowest PAR denoted by ${\underline{S^{\prime}}}^{f}$ is the selected vector to be transmitted. In the proposed system, modified SLM scheme is applied which is more effective than the ordinary in which the length of the vectors $\left\{P^{(g)}\right\}$ is reduced to be $N / F_{d}$ where $F_{d}=2^{j} \in\{2,4,8,16, \ldots$ etc $\}$ then only certain elements of $\underline{S}^{f}$ are selected to be multiplied by those vectors elements whereas, the rest items remain unchanged as follows:

$$
S_{i}^{f}(g)=S_{i}^{f} \cdot P_{j}^{(g)} \quad \cdots
$$

where $_{i=0}, \frac{N}{F_{d}}-1, \frac{2 N}{F_{d}}-1, \quad \ldots \quad N-1$

$j=1,2, \quad \ldots \quad F_{d}$

Here, the number of multiplications will be smaller than in the ordinary SLM technique.

\subsection{Partial Transmit Sequence (PTS) Algorithm}

In the traditional PTS scheme [7], a group of complex exponential vectors $\left\{b^{(v)}\right\}$ are randomly generated with length $N$ and the vector terms are given by $b^{(v)}=e^{j \theta_{v}}, 1 \leq v \leq V$. The set of phases $\left\{\theta_{\mathrm{v}}\right\}$ are randomly selected from the range $[0,2 \pi$ [. Each vector is multiplied by the vector $\underline{S}^{f}$ giving a group of vectors $\left\{\underline{S}^{f}(v), 1 \leq v \leq V\right\}$ which could be represented as follows:

$$
S_{n}^{f}(v)=S_{n}^{f} \cdot b_{n}^{(v)} \quad, \quad 0 \leq n \leq N-1 \quad \cdots
$$

And similarly, the PAR is calculated for all obtained vectors and the one with the lowest PAR denoted by ${\underline{S^{\prime}}}^{f}$ is the selected vector to be transmitted. But in this paper, a modified PTS technique [1] is applied in which the vector length of $\left\{b^{(v)}\right\}$ is reduced to be of only N/C items where $C=2^{j} \in\{2,4,8,16, \ldots$ etc $\}$. The difference between ordinary and modified PTS scheme is exactly as in the case of SLM approach, where certain elements of $\underline{S}^{f}$ is multiplied by those vectors elements whereas, the rest items remain unchanged as follows:

$$
S_{i}^{f}(v)=S_{i}^{f} \cdot b_{j}^{(v)} \quad \cdots
$$

where $_{i=0}, \frac{N}{C}-1, \frac{2 N}{C}-1, \ldots N-1 \quad, \quad j=1,2, \ldots \quad C$

\subsection{Space Time Block Coder (STBC)}

In multi-transmitting antennas system, instead of emitting the same symbol by all antennas at the same time, $L$ successive symbols (over $L$ signaling intervals) are arranged to be transmitted through $N_{t}$ transmitting antennas by a certain order and this is the main concept of 
STBC system[2]. In the proposed system, two cases are considered; two transmitting and four transmitting antennas cases ( $N_{t}=2$ and $N_{t}=4$ respectively).

\section{Two transmitting antennas:}

In this case two successive symbols $\mathrm{S}_{1}$ and $\mathrm{S}_{2}$ are stored in the STBC encoder memory. At first signaling interval, antennal emits symbol $S_{1}$ and antenna 2 emits symbol $S_{2}$. Then at second signaling interval, antennal emits $-S_{2}^{*}$ whereas, antenna 2 emits $S_{1}^{*}$. This can be expressed in matrix form as follows:

$$
\left[\begin{array}{cc}
S_{1} & -S_{2}^{*} \\
S_{2} & S_{1}^{*}
\end{array}\right] \quad \cdots
$$

\section{Four transmitting antennas:}

The symbol transmission arrangement could be expressed in matrix form as follows:

$$
\left[\begin{array}{cccc}
S_{1} & -S_{2} & -S_{3}^{*} & -S_{4}^{*} \\
S_{2} & S_{1} & S_{4}^{*} & -S_{3}^{*} \\
S_{3} & -S_{4} & S_{1}^{*} & S_{2}^{*} \\
S_{4} & S_{3} & -S_{2}^{*} & S_{1}^{*}
\end{array}\right] \ldots
$$

In this case there are four symbols to be transmitted in four successive signaling intervals using four transmitting antennas. The column number denotes the antenna number

where; the row number denotes the signaling time interval number.

\subsection{Preamble Design for Multiple - Antenna OFDM-CDMA}

The preamble is a complex vector $\left\{X_{i}, 1 \leq i \leq N_{t}\right\}$ added at each transmitting antenna before real transmission of data which acts as a pilot sequence helps the receiver in channel estimation process. Those vectors are designed to be orthogonal in order not to have interference between those vectors at each receiving antenna, and they should be independent in order to monitor fading channel parameters at the receiver. The selected phases for the preambles depend on two things; the number of transmitting antennas $N_{t}$ and the number of IFFT points. The preamble vector can be displayed in matrix notation as follows:

$$
\underline{X}=\left[\begin{array}{c}
\underline{X}_{1} \\
\underline{X}_{2} \\
\cdot \\
\cdot \\
\cdot \\
\underline{X}_{N_{t}}
\end{array}\right]=\left[\begin{array}{c}
e_{1} \underline{X}_{1} \\
e_{2} \underline{X}_{1} \\
\cdot \\
\cdot \\
\cdot \\
e_{N_{t}} \underline{X}_{1}
\end{array}\right] \quad \ldots
$$

where; $\underline{X}_{1}=\left[\begin{array}{lllll}1 & 1 & 1 & & 1\end{array}\right], e_{i}=\exp \left(j \frac{2 \pi n p_{i}}{N}\right), n=0,1,2, \ldots, N-1$

$$
p_{i}=\frac{(i-1) N}{N_{t}} \text { and } .=1,2, \ldots, N_{t}
$$

Fig.2 displays an example of frequency domain preamble vectors with $N_{t}=4$ and $N=8$ 

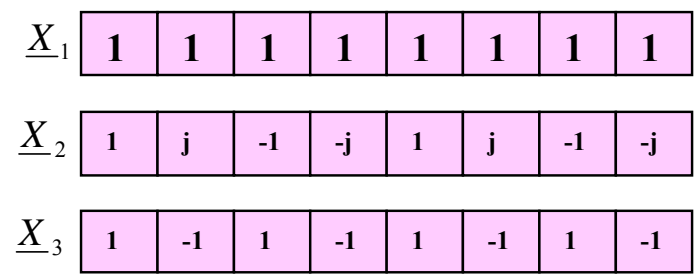

$\underline{X}_{4}$\begin{tabular}{|l|l|l|l|l|l|l|l|}
\hline 1 & $-\mathbf{j}$ & -1 & $\mathbf{j}$ & $\mathbf{1}$ & $-\mathbf{j}$ & -1 & $\mathbf{j}$ \\
\hline
\end{tabular}

Fig. 2 Preamble Vectors in Frequency Domain with $N_{t}=4$ and $N=8$.

Following to those preambles transmission there should be interval of no signaling, called guard interval (GI), in order to mitigate any interference that may happen between two successive signaling intervals. Therefore the GI is chosen to be greater than or equal the coherence time of the fading channel or by another word $N_{G I} \geq N_{P}$ where; $N_{G I}$ is the number of chips equivalent to the guard interval and $N_{P}$ is the maximum number of paths between any pair of transmitting and receiving antennas.

\section{Channel Model}

The channel between each pair of transmitting and receiving antennas is a multipath fading channel. The maximum number of paths between any transmitting and receiving antennas pair is denoted by $N_{P}$ each path contains $N$ coefficients with complex Gaussian distribution (Rayleigh envelope). The fading is assumed to be of frequency selective fast type. The impulse response of the channel between the transmitting antenna $i$ and the receiving antenna $j$ could be expressed as follows:

$$
h_{i j}=\left[\begin{array}{lllll}
h_{i j}(0) & h_{i j}(1) & h_{i j}(2) & \ldots & h_{i j}\left(N_{P}-1\right)
\end{array}\right] \quad \ldots
$$

By evaluating N-point FFT for the channel impulse response given in equation (12), we will get the channel frequency response as follows:

$$
H_{i j}=\left[\begin{array}{lllll}
H_{i j}(0) & H_{i j}(1) & H_{i j}(2) & \ldots & H_{i j}(N-1)
\end{array}\right] \quad \ldots
$$

The frequency response parameters of the channel between any transmitting and receiving antennas $\left\{H_{i j} \quad 1 \leq i \leq N_{t}, \quad 1 \leq j \leq N_{r}\right\}$ are assumed to be orthogonal and independent.

\section{STBC OFDM - CDMA Receiver Model}

The receiver model is illustrated at Fig. 3 and the stages of reception process will be displayed in the coming subsections.

\subsection{Multi - Channel Separation and Channel Estimation}

If the preamble is designed in efficient way, as mentioned in equation (11), the convolution between it and the channel impulse response will be as follows:

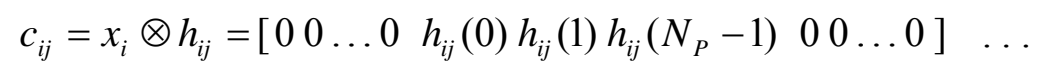

$$
\begin{aligned}
& 6 / 16
\end{aligned}
$$


This means that, the convolution between the preamble emitted from transmitting antenna $i$ and the coefficients of the channel between transmitting antenna $i$ and receiving antenna $j$, will result in the actual coefficients of that channel without any interfering parameters from other paths. The received pilot signal vector at the $j^{\text {th }}$ receiver antenna could be expressed as follows:

$$
r_{j}=\sum_{i=1}^{N_{t}} c_{j i}+n_{j} \quad \ldots
$$

where $n_{j}$ is an additive white Gaussian noise (AWGN) vector with zero mean and variance $\sigma_{n}^{2}$.
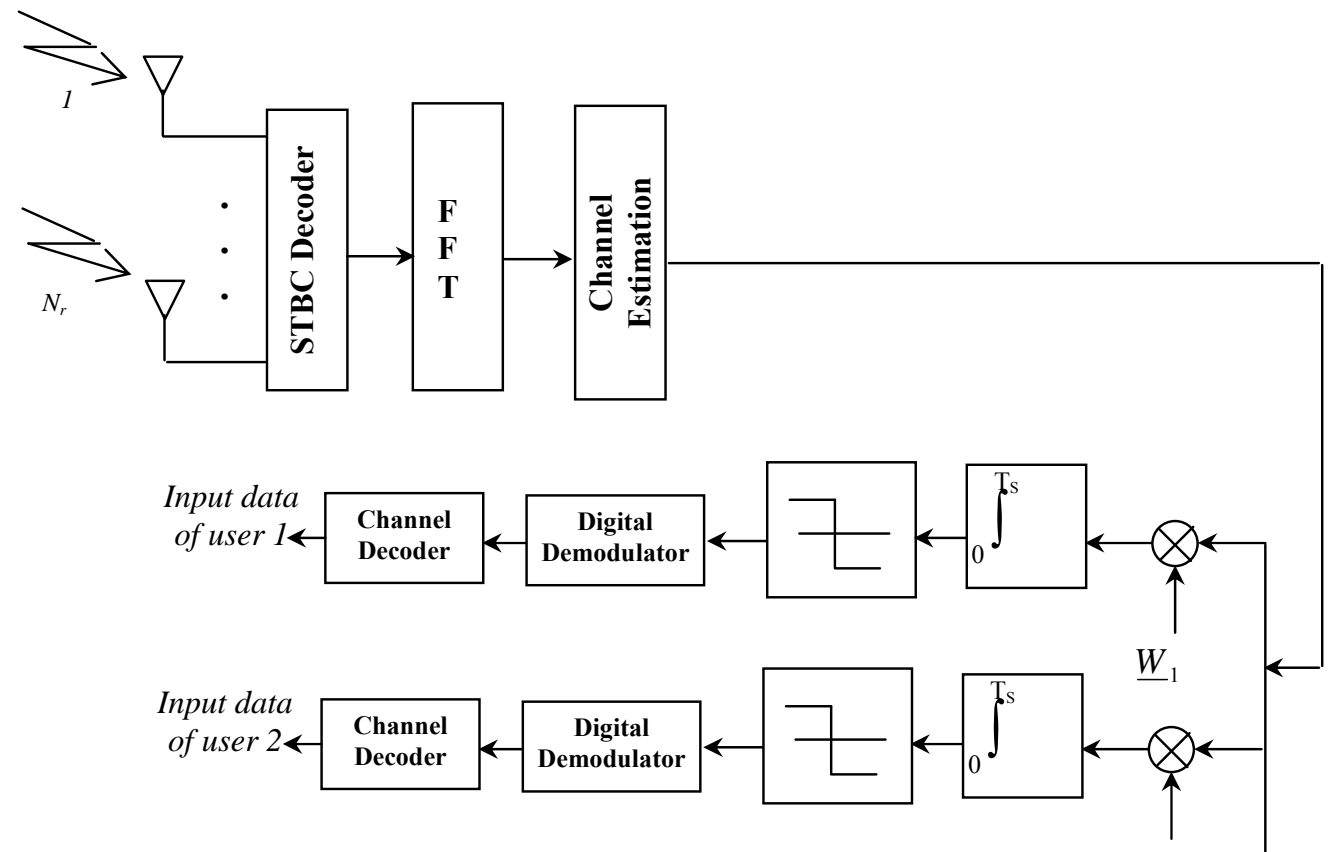

$\underline{W}_{2}$
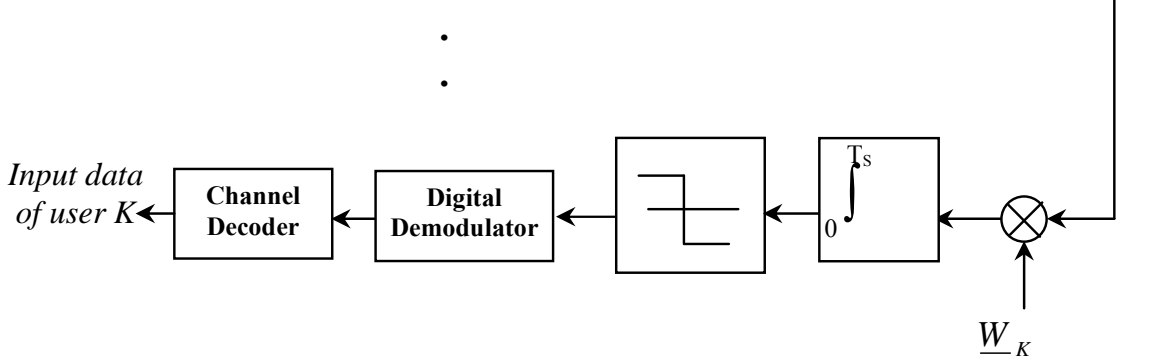

Fig. 3 "STBC OFDM - CDMA Receiver Model". 
Each term denoted by $c_{j i}$ contains $N_{P}$ components which represents the paths coefficients included in that channel. Fig. 4 displays in graphical form all coefficients seen by receiving antenna $j$ denoted by $\underline{C}_{j}$.

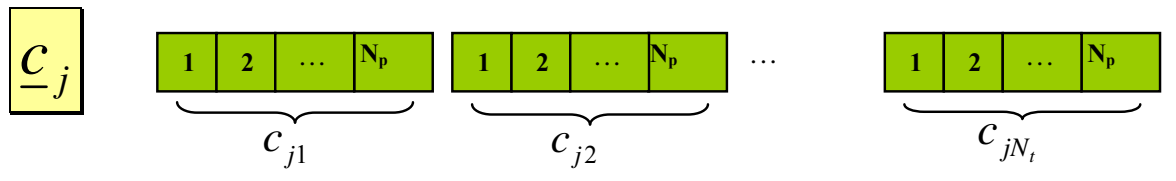

Fig. 4 Channel coefficients vector seen by receiving antenna $\mathbf{j}$.

In order to separate each vector $\underline{c}_{j i}$, belong to each transmitting receiving antenna pair, windowing scheme is used here. In such scheme, the received vector $\underline{r}_{j}$ is multiplied by a rectangular function $\Lambda$ with width $S_{W}=\left\lfloor\frac{N}{N_{t}}\right\rfloor$ which in ideal case should equal $N_{P}$. The multiplication operation will result in the vector $\underline{r}_{j}$ which could be expressed as follows:

$$
\underline{r}_{j}^{\prime}=\Lambda r_{j}=c_{j}+n_{j}^{\prime}=\left(x \otimes h_{j}\right)+n_{j}^{\prime} \quad \ldots
$$

where;

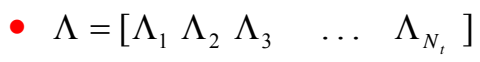

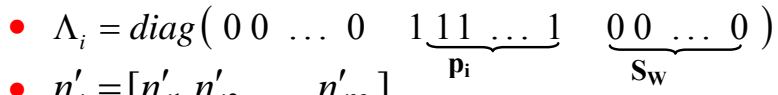

- $n_{j}^{\prime}=\left[\begin{array}{llll}n_{j 1}^{\prime} & n_{j 2}^{\prime} & \ldots & n_{j N_{t}}^{\prime}\end{array}\right]$

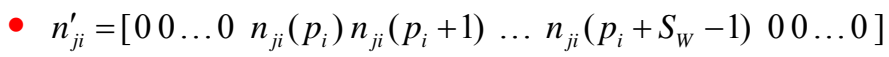

By applying FFT into both sides of equation (16), we can obtain the received pilot signal vector, seen by receiving antenna $j$, in the frequency domain as follows:

$$
R_{j}=X H_{j}+N_{j} \quad \ldots
$$

where;

- $\mathrm{X}$ : is the preamble matrix (frequency domain) $=\operatorname{diag}\left(\begin{array}{llll}X_{1} & X_{2} & \ldots & X_{N_{t}}\end{array}\right)$

- $H_{j}$ : is channel frequency response coefficients seen by receiving antenna $\mathrm{j}=$ $\operatorname{diag}\left(H_{j 1} H_{j 2} \quad \ldots \quad H_{j N_{t}}\right)$ and $H_{j i}=F F T\left(h_{j i}\right)$

- $N_{j}$ : is the AWGN matrix seen by receiving antenna $\mathrm{j}$ in the frequency domain = $\operatorname{diag}\left(N_{j 1} N_{j 2} \quad \ldots \quad N_{j N_{t}}\right)$ and $N_{j i}=\operatorname{FFT}\left(n_{j i}\right)$

As observed from equations (15) and (17), the pilot preamble is vital in channel parameters estimation process therefore, they should be designed carefully [9] and [10]. At the receiver, the vector $R_{j}$ is multiplied by the matrix $X^{-1}$ resulting in the vector $\widetilde{H}_{j}$ which contains an estimate for the channel frequency response as follows:

$$
\widetilde{H}_{j}=H_{j}+X^{-1} N_{j} \quad \cdots
$$




\subsection{Real Data Transmission}

The frequency domain received vector received by receiving antenna $\mathrm{j}$ (obtained after $\mathrm{N}-$ point FFT operation) will be as follows:

$$
R_{j}=S^{f} \sum_{i=1}^{N_{t}} H_{i j}+N_{i j} \quad \ldots
$$

where; $S^{f}$ is the frequency domain transmitted data vector in the final form (after symbol formatting and applying PAR reduction scheme). The received vector is then multiplied by the inverse of the estimated channel matrix as follows:

$$
\widetilde{S}=\widetilde{H}^{-1} R_{j} \quad \cdots
$$

After that, the vector $\widetilde{S}$ is applied into the dispreading process to give a set of observation items denoted by $\left\{Z_{k}, 1 \leq k \leq K\right\}$ from which the transmitted symbols by each user can be estimated by making threshold detection. The dispreading process could be expressed as follows:

$$
Z_{k}=\sum_{n=1}^{N} \widetilde{S}(n) \cdot W_{k}(n) \quad \ldots
$$

\section{Performance Evaluation and Simulation}

The STBC OFDM - CDMA system has been simulated using computer software and the results are displayed in form of curves and tables. The man objective of any digital communication system is to reduce the bit error rate (BER) as much as possible, so most of the coming figures represent the effect of different system parameters on the BER. For all the coming results, we have assumed that $M=N$ where $\mathrm{M}$ is the number of $\mathrm{PN}$ code chips and $\mathrm{N}$ is the number of FFT points.

\subsection{The Effect of Each PAR Reduction Scheme}

The first set of curves shown in Fig. 5 displays the effect of each scheme used in PAR reduction on the calculated PAR level at different signaling interval. As shown in the figure, the lowest PAR level is obtained when applying the three schemes together. But the simulation showed that symbol formatting has the largest effect on reducing the PAR whereas, the PTS scheme causes the PAR to be reduced by a little value.

\subsection{The Effect of SLM Approach Parameters}

In the next set of curves, we have focused on one of the SLM approach parameters denoted by $\mathrm{F}_{\mathrm{d}}$. Figure 6 displays the PAR level at different values of the processing gain $\mathrm{M}$ value. As seen by the shown curves, the PAR increases with the processing gain increment. Whereas, the PAR level is reversely proportional to the value $F_{d}$. The effect of the parameter $\mathrm{G}$ used in the SLM approach is very small and simulation showed that any value for $\mathrm{G}>2$ will result in the same PAR level. 


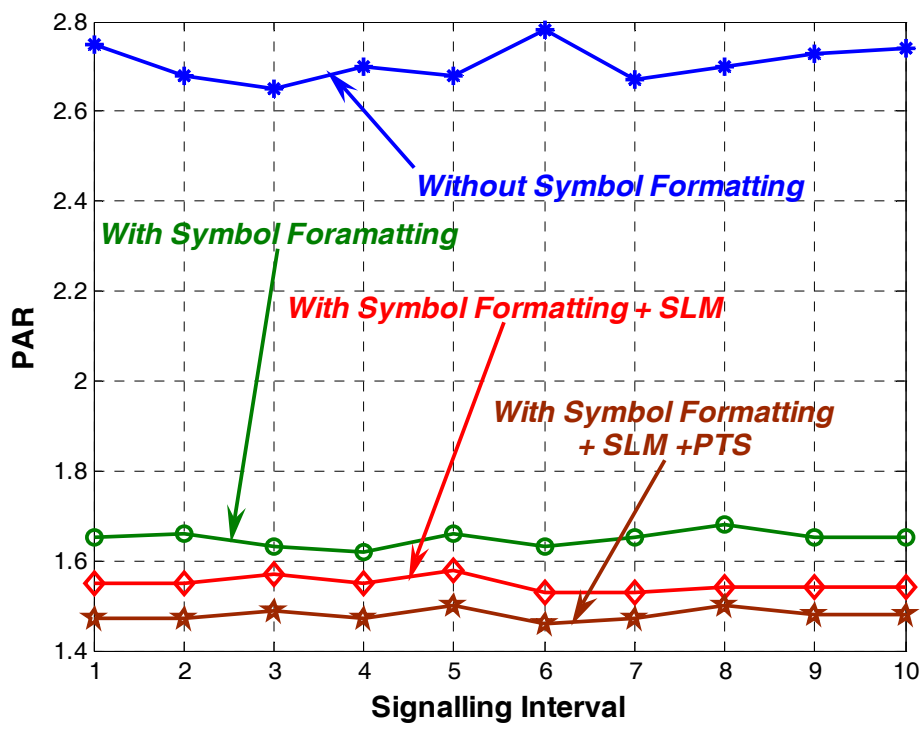

Fig. 5 PAR level at different signaling intervals with $\mathbf{G}=\mathbf{3 0}$, $\mathrm{Fd}=16, \mathrm{~K}=10, \mathrm{Nt}=1, \mathrm{Nt}=1$, and $\mathrm{M}=32$.

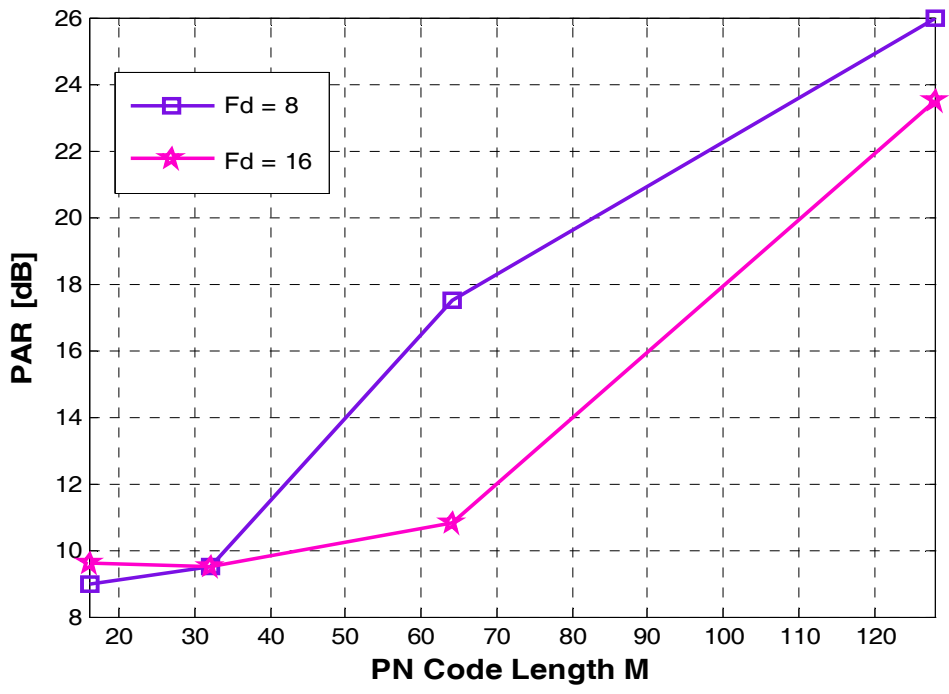

Fig. 6 PAR level versus variation in $M$ value with $\mathrm{Nt}=1, \mathrm{Nr}=1$, $\mathrm{G}=10$, and $\mathrm{K}=7$. 


\subsection{The Effect of the Processing Gain on the System Performance}

Beginning from this subsection, a complete STBC OFDM - CDMA system has been simulated. Figure 7 displays the BER performance versus variation in the SNR, assuming equal SNR for all the $\mathrm{K}$ users, at three cases $\mathrm{M}=16,32$, and 64 . Although the increment of $\mathrm{M}$ causes increment in the PAR level (as mentioned before) that increment results in reduction in the BER by a remarkable way. At $\mathrm{SNR}=5 \mathrm{~dB}$ the obtained BER at $\mathrm{M}=16,32$, and 64 are $0.15,0.04$, and 0.0012 respectively. At that set of curves, the system is simulated without any of channel coding schemes.

\subsection{The Effect of Applying Channel Coding Technique on the BER Level}

It is well known that by applying any of the channel coding techniques; very low bit error rate could be obtained because of its ability of error detection and correction at the receiver. This is clearly illustrated at Fig.8 which displays the BER performance against variation of SNR at three situations; no channel coding, using Hamming coding, and using Convolutional coding. The applied Hamming Encoder is of 4 bits input and 7 bits output. The Convolutional encoder is shown at Fig. $(15-a)$.

\subsection{The Effect of the Number of Paths on the System Performance}

The number of fading paths has destructive effect on the received signal power. By another word, the greater is the number of fading paths, more scattering will be the transmitted signal. This well appears at Fig.9 which represents the BER performance versus variation in the SNR at four values for the paths number; $2,3,6,10$. The simulation results showed that the BER increases with the increment in the number of paths.

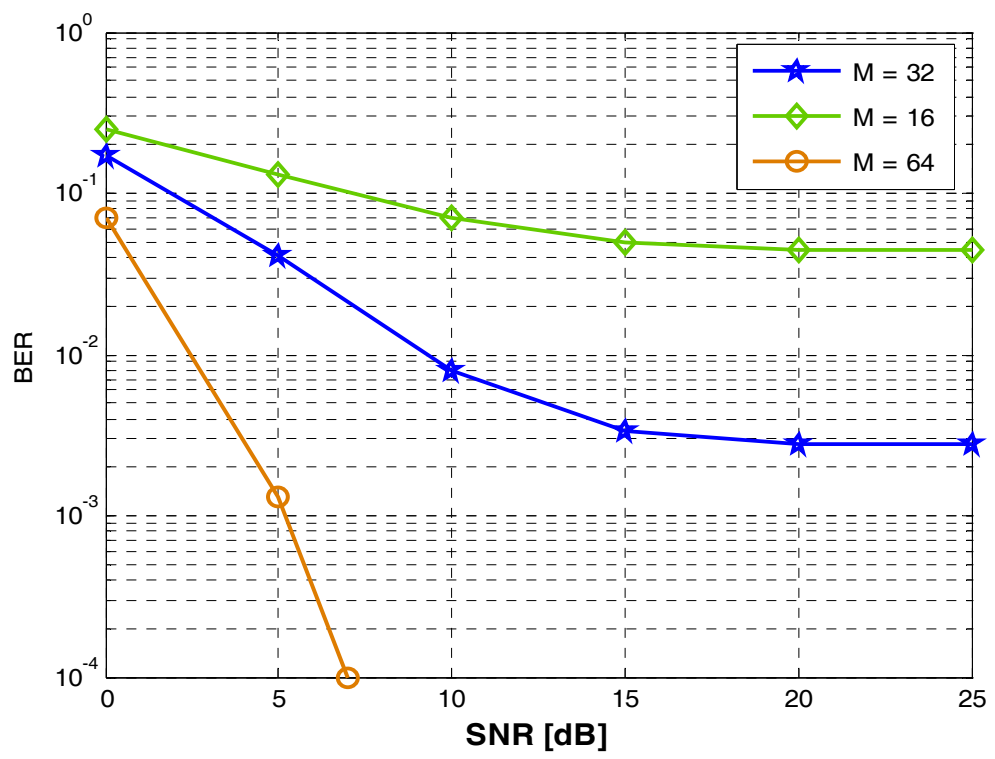

Fig. 7 BER performance versus variation of SNR, assuming equal SNR for all users, with following system parameters: $\mathrm{Nt}=1, \mathrm{~Np}=3, \mathrm{~K}=10$, and $\mathrm{Fd}=8$. 


\subsection{The Effect of the Number of Transmitting antennas on the System Performance}

Since the transmitted signals by the $N_{t}$ transmitting antennas are summated at each receiving antenna, the increment in the number of transmitting antennas $N_{t}$ results in increment in the received power which enhances the BER performance (causes reduction in the BER). In Fig.10, the BER performance is displayed versus variation in the SNR at three cases; $\mathrm{N}_{t}=1$, 2 , and 4 . The coming table contains the BER obtained at $\mathrm{SNR}=4 \mathrm{~dB}$.

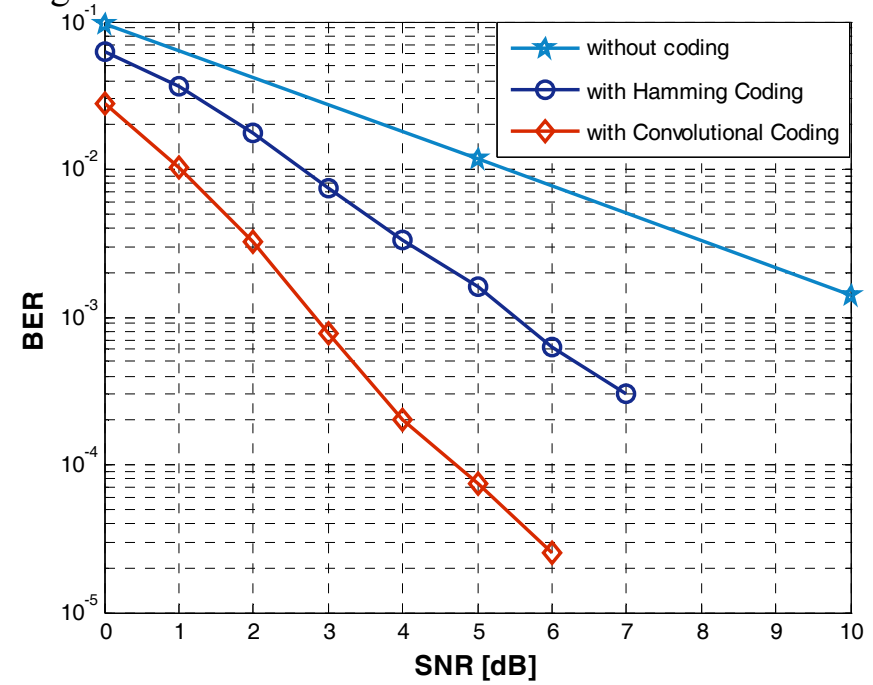

Fig. 8 BER performance versus variation of SNR, assuming equal SNR for all users, with following system parameters:

$\mathrm{Nt}=2, \mathrm{Nr}=1, \mathrm{~Np}=3, \mathrm{~K}=10, \mathrm{M}=32, \mathrm{G}=30$, and $\mathrm{Fd}=8$.

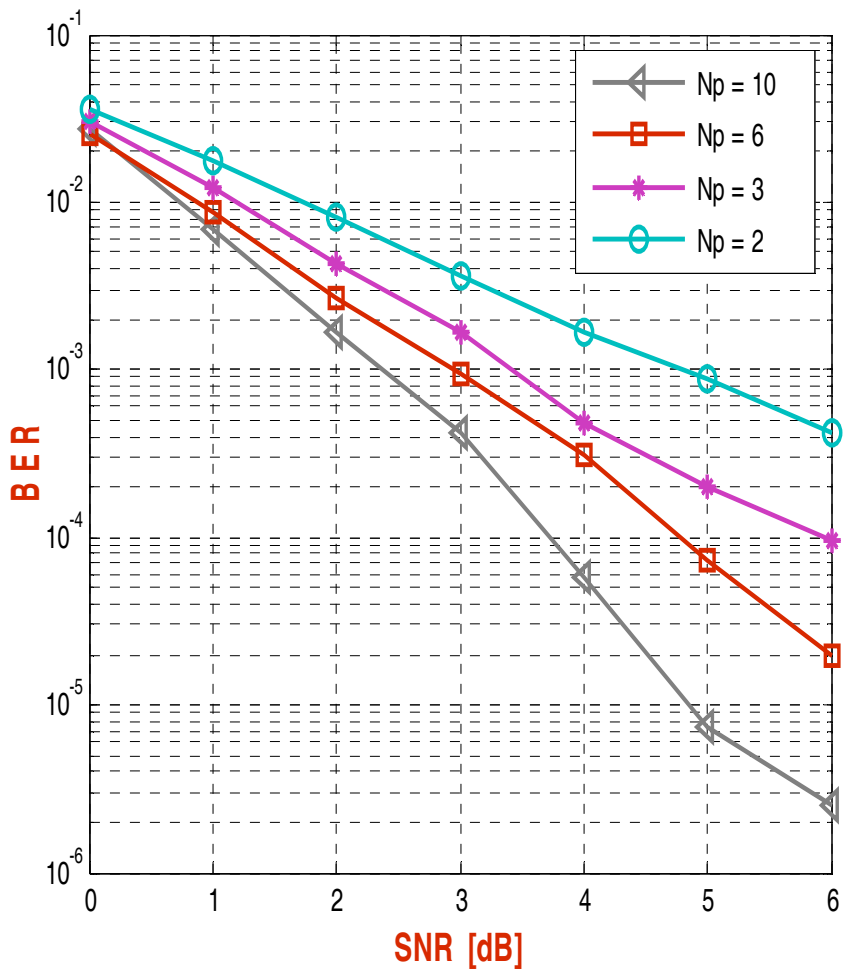

Fig. 9 BER performance versus variation SNR of the proposed system with Convolutional coding, assuming equal SNR for all users, with following system parameters: $\mathrm{Nt}=2, \mathrm{Nr}=1, \mathrm{G}=30, \mathrm{M}=32, \mathrm{~K}=10$, and $\mathrm{Fd}=8$. 


\subsection{The Effect of the Modulation Technique on the System Performance}

When selecting the modulation technique, we should trade off between the hardware complexity and the performance efficiency. Take for example three of digital modulation techniques; BPSK, DBPSK, and QPSK, the simplest technique is the BPSK then the DBPSK and the QPSK is the most complicated technique but when talking about the BER, the sorting order will be reversed i.e. the QPSK results in the lowest BER then the DBPSK and BPSK have higher BER. This is shown at Fig. 11 which represents the BER performance versus SNR variation using the three modulation techniques mentioned before.

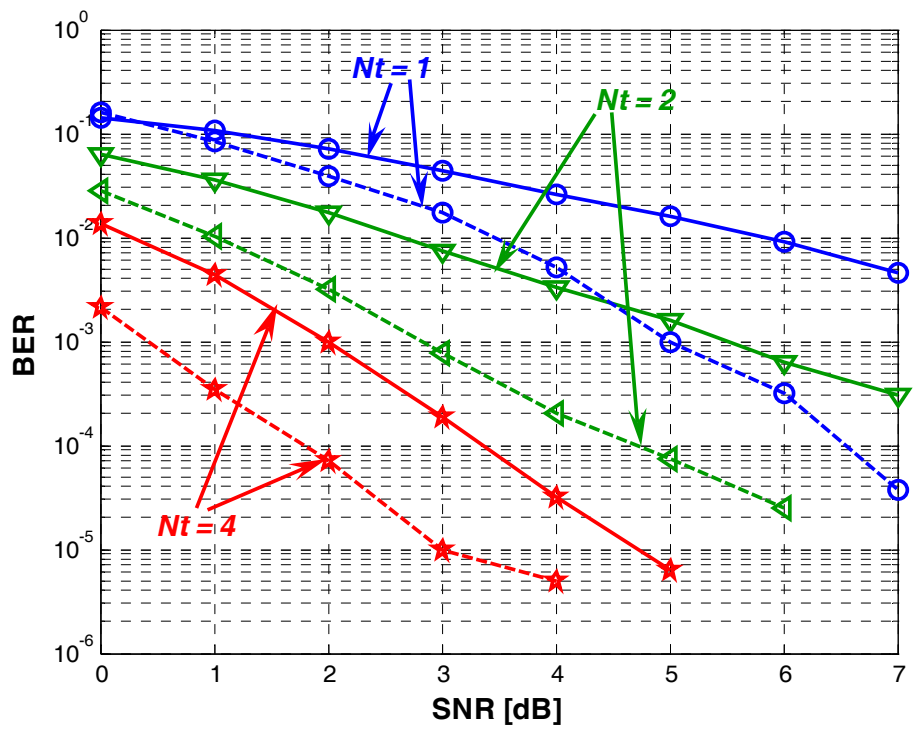

Fig. 10 BER performance versus variation in the number of users $K$, assuming equal SNR for all users, with following system parameters: $\mathrm{Nr}=1, \mathrm{~Np}=3, \mathrm{~K}=10, \mathrm{G}=30$, and

$\mathbf{F d}=8$. (Solid line for Hamming coding, dashed line for Convolutional coding).

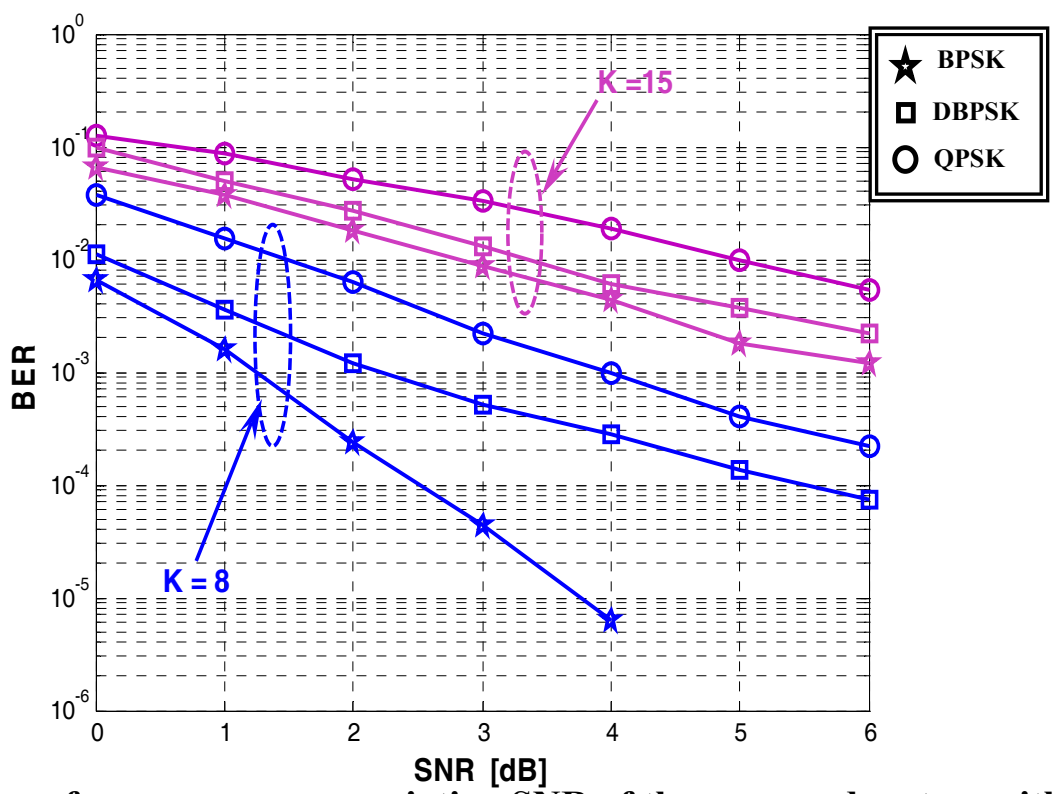

Fig. 11 BER performance versus variation SNR of the proposed system with Hamming coding, assuming equal SNR for all users, with following system parameters: $\mathrm{Nt}=\mathbf{2}$, $\mathrm{Nr}=1, \mathrm{G}=30, \mathrm{M}=32$, and $\mathrm{Fd}=8$. 


\subsection{The Effect of Convolutional Encoder Design on the System Performance}

Figure 12 displays the BER performance against variation in the number of users $\mathrm{K}$ using different designs for the Convolutional Encoder and those four encoders are shown in figures $13,14,15$, and 16 . There are two factors to be considered when designing convolutional encoder; the number of shift registers, and the number of outputs. Simulation results showed that the greater is the number of shift registers or the number of outputs, the lower will be the BER level.

\subsection{The Effect of Hamming Encoder Design on the System Performance}

The Hamming coding technique could be applied as an example of channel block coding techniques. It is simpler in execution than the convolutional coding since it can be executed in software manner using the suitable generator matrix. The shown set of curves at Fig.17 displays the BER performance versus variation in the number of users at three coder designs in two cases; $\mathrm{N}_{\mathrm{t}}=2$ and $\mathrm{N}_{\mathrm{t}}=4$. In table I a comparison among the three coder designs and the obtained BER at $\mathrm{K}=10$ and $\mathrm{N}_{\mathrm{t}}=4$.

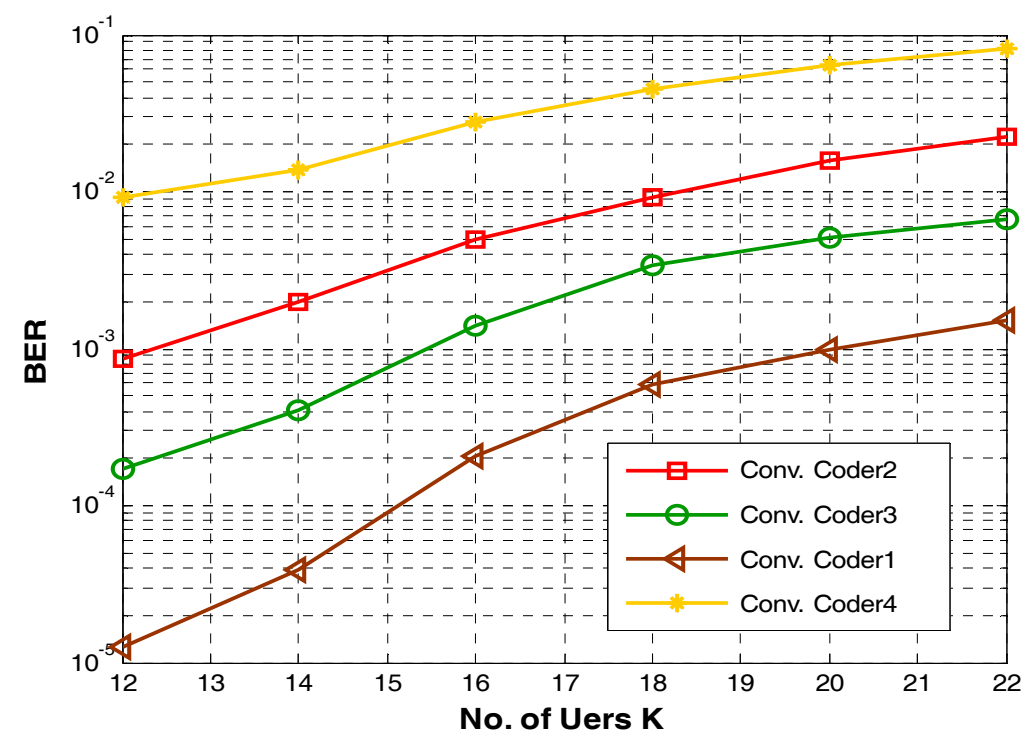

Fig. 12 BER performance versus variation in the number of users $\mathrm{K}$ with convolutional coding $\mathrm{G}=30, \mathrm{SNR}=4 \mathrm{~dB}, \mathrm{Fd}=8, \mathrm{Nt}=2, \mathrm{Nr}=1$, and $\mathrm{Np}=3$.

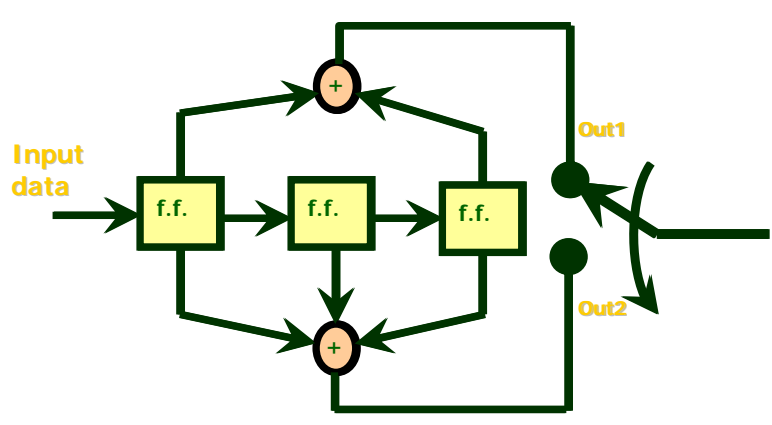

Fig. 13 Convolutional Coder1.

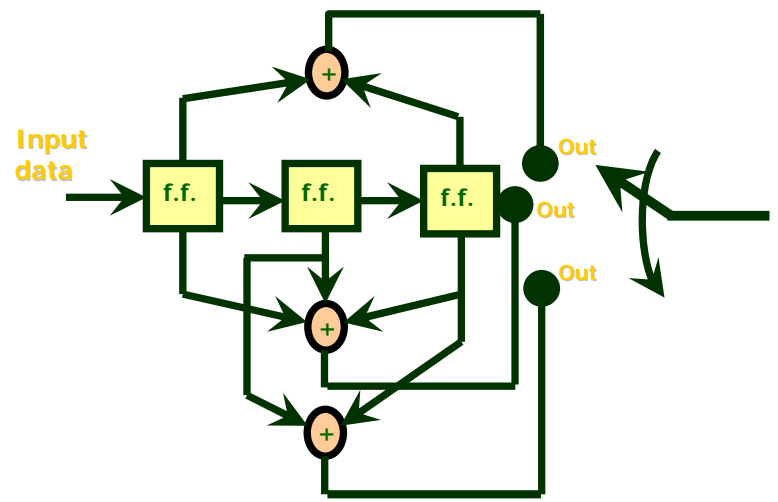

Fig. 14 Convolutional Coder2. 


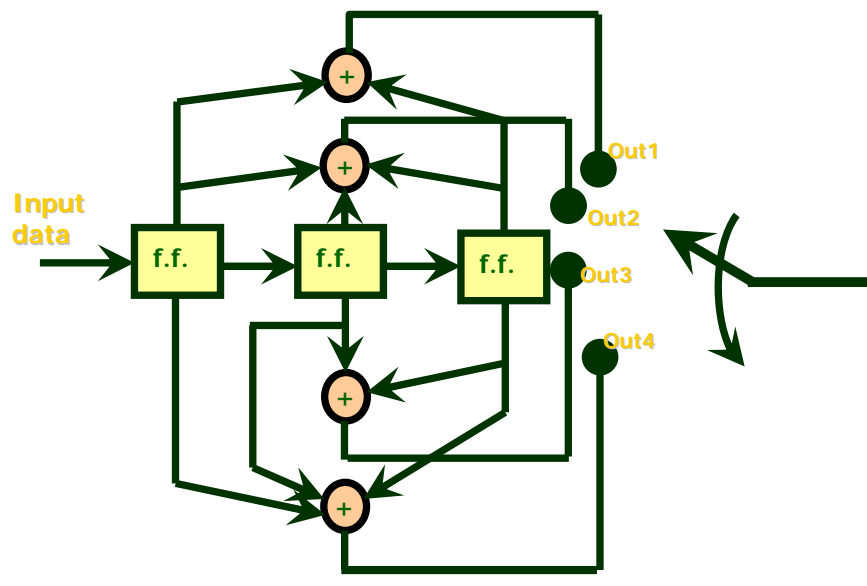

Fig. 15 Convolutional Coder3.

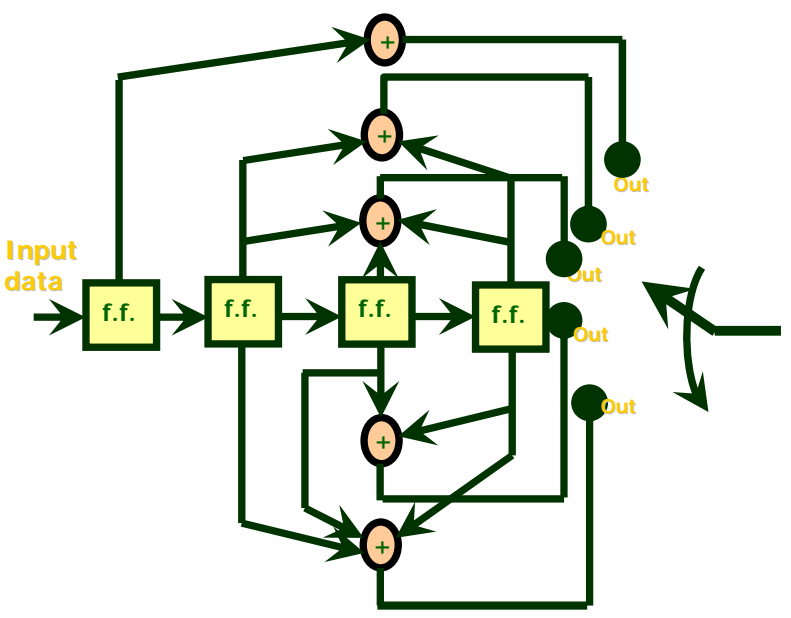

Fig. 16 Convolutional Coder4.

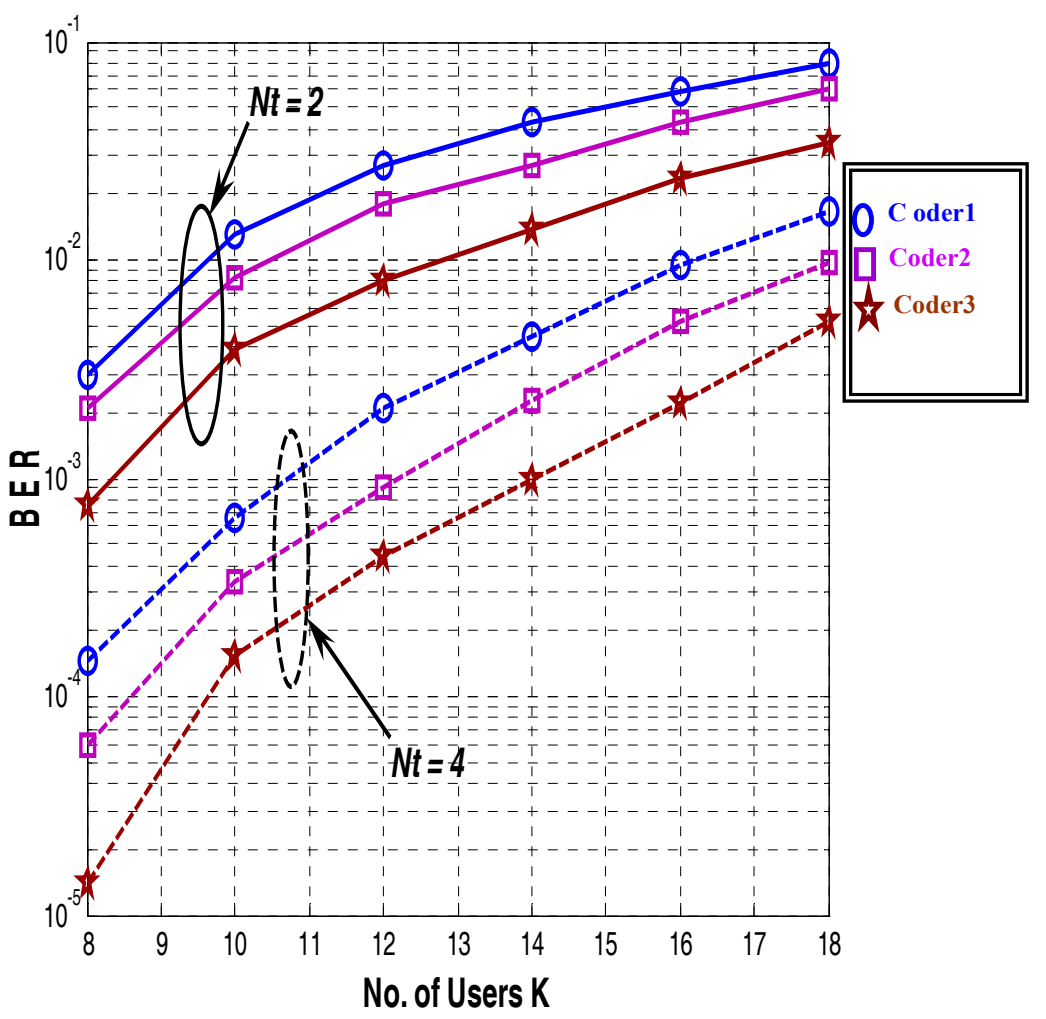

Fig. 17 BER performance versus variation in the number of users $\mathrm{K}$ with Hamming coding $\mathrm{G}=30, \mathrm{SNR}=4 \mathrm{~dB}, \mathrm{Fd}=8, \mathrm{Nt}=2, \mathrm{Nr}=1$, and $\mathrm{Np}=3$.

Table 1

\begin{tabular}{|c|c|c|c|}
\hline \hline Encoder Design & Data - to - Code length & Code Efficiency & BER \\
\hline \hline Coder1 & $26-$ to -31 & $83.87 \%$ & $6 \times 10^{-4}$ \\
\hline Coder2 & $11-$ to -15 & $73.33 \%$ & $3 \times 10^{-4}$ \\
\hline Coder3 & $4-$ to -7 & $57.14 \%$ & $1.5 \times 10^{-4}$ \\
\hline
\end{tabular}




\section{Conclusion}

The main function of the proposed STBC OFDM- CDMA system is to mitigate the distortion caused by the worst type of fading which is the fast frequency selective fading. Actually the secret is that the OFDM system can convert the fast frequency selective fading into flat fading whereas, the DS-CDMA system converts the fast fading into slow fading channel and this new form of fading has less distortion effect than the actual type. The system simulation using computer software showed that the BER has reached satisfying level. By adding any of channel coding techniques, the BER is reduced to the order of $10^{-6}$ at $\mathrm{SNR}=4 \mathrm{~dB}$. But there are two disadvantages of the proposed system; the first is the necessity of using PAR reduction schemes with the OFDM systems which causes reduction in the SNR measured at the transmitter. The second disadvantage is the need for preamble transmission before transmitting each symbol in order to estimate the channel at the receiver resulting slowing down the transmission rate.

\section{References}

[1]. You et al, "Performance Evaluation of OFDM-CDMA with Multiple Antennas for Broadband Wireless Networks", IEEE TRANSACTION ON VEHICULAR TECHNOLOGY, Vol. 54, No.1, January 2005.

[2]. Jankiraman, "Space-Time Codes and MIMO Systems", Mohinder Jankiraman, 2004.

[3]. Yeh and Y. Lin, "Channel estimation using pilot tones in OFDM systems", IEEE Trans. Broadcast, vol. 45, no. 4, pp. 400-409, Dec. 1999.

[4]. D. Agarwal, V. Tarokh, A. Naguib, and N. Seshadri, "Space-time coded OFDM for high data rate wireless communication over wide-band channels" in Proc. VTC'98, May 1998, pp. 2232-2236.

[5]. S. H. Muller and J. B. Huber, "OFDM with reduced peak-to-average power ratio and optimum combination of partial transmit sequences", Electron, Lett., vol. 30, no. 25, pp. 2098-2099, 1994.

[6]. R. W. Bauml, R. F. H. Fischer, and J. B. Huber, "Reducing the peak-to-average power ratio of multicarrier modulation by selected mapping", Electron, Lett., vol. 32, no. 22, pp. 2056-1257, 1996.

[7]. N. Ruangsrat and R. M. A. P. Rajatheva, "An investigation of peak-to-average power ratio in MC-CDMA combined with partial transmit sequence", in Proc. VTC'01, May 2001, pp. 6-9.

[8]. N. Ohkubo and T. Ohtsuki, "A peak to average ratio reduction of multicarrier CDMA using selected mapping", in Proc. VTC'02, Sep. 2002, pp. 2086-2090.

[9]. Y. Li, N. Seshadri, and S. Ariyavisitakul, "Channel estimation for OFDM systems with transmitter diversity in mobile wireless channels", IEEE J. Sel. Areas Comm. Vol. 17, no. 3, pp. 461-471, Mar. 1999.

[10]. Y. Li, "Simplified channel estimation for OFDM systems with multiple transmit antennas", IEEE Trans. Wireless Comm., vol. 1, no. 1, pp. 67-75, Jan. 2002. 